Copyright (C) 2014 IEEE. Personal use of this material is permitted. Permission from IEEE must be obtained for all other uses, in any current or future media, including reprinting/republishing this material for advertising or promotional purposes, creating new collective works, for resale or redistribution to servers or lists, or reuse of any copyrighted component of this work in other works. 


\title{
Variable Fractional Delay FIR Filter Design with Bi-criteria and Coefficient Relationship
}

\author{
Hai Huyen Dam
}

\begin{abstract}
This paper investigates a trade-off between the integral squared error and the peak deviation error for a variable fractional delay (VFD) filter with coefficient relationship. The integral squared error is minimized subject to additional constraints on the peak deviation error. The problem is solved by utilizing the second-order cone programming (SOCP). In addition, the performance of the VFD filter with discrete coefficients is investigated where the filter coefficients are expressed as the sum of power-oftwo terms to reduce the filter operations to shifts and adds. Design examples show that the peak deviation error can be reduced significantly from the least squares solution while maintaining approximately the same integral squared error. Similarly, the integral squared error can be reduced significantly from the minimax solution while maintaining approximately the same peak deviation error. Also, the trade-off filters are less sensitive with respect to quantization than the least squares and minimax solutions.
\end{abstract}

\section{INTRODUCTION}

Digital filters with tunable fractional phase-delay or fractional group delay, referred to as variable fractional delay (VFD) filters, are useful in various signal processing applications [1]-[8]. A range of applications have been considered, including timing offset recovery in digital receivers, comb filter design, sampling rate conversion, speech coding, time delay estimation, one-dimensional digital signal interpolation and image interpolation. The VFD filters belong to a branch of variable digital filters that are applicable in applications in which the frequency characteristics need to be adjustable, and are generally implemented by the Farrow structure [1], in which a parameter is used to control the delay online without redesigning a new filter.

In [6]-[7], a symmetric/antisymmetric coefficient relationship is developed for VFD FIR filter coefficients to reduce the number of design coefficients to about half of the original design. In [8], another coefficient relationship is introduced to enable the number of design coefficients to be further reduced by half. In all cases, the VFD filters are designed using a least squares criterion [7]-[8] or a minimax criterion [9]-[10]. The least squares filter in general has a low integral squared error and a large peak deviation error. The minimax filter, on the other hand, has a low peak deviation error with a large integral squared error.

Hai Huyen Dam is with Department of Mathematics and Statistics, Curtin University of Technology, Kent Street, Bentley, Perth, WA 6102, Australia. Email H.Dam@curtin.edu.au. Phone: +6189266 7670, Fax: +6189266 3197 .

This research was supported by ARC Discovery Project DP120103859.

Copyright (c) 2013 IEEE. Personal use of this material is permitted. However, permission to use this material for any other purposes must be obtained from the IEEE by sending an email to pubspermissions@ieee.org
As such, it is important to find a trade-off between the integral squared error and the peak deviation error.

In this paper, we investigate the design of VFD filters with minimum integral squared error subject to constraints on the peak deviation error from the desired response. The obtained filters are compared with the least squares and minimax solutions. We show that a trade-off curve can be achieved between the integral squared error and the peak deviation error by having constraints on different levels of the peak deviation error. The least squares and the minimax solutions are the two extreme points in the trade-off curve. The integral squared error can be reduced significantly from the minimax solution while maintaining approximately the same peak deviation error. Similarly, the peak deviation error can be significantly reduced from the least squares solution while maintaining approximately the same integral squared error. In addition, the performance of the VFD filter with discrete coefficients is investigated where an efficient quantization scheme is employed to distribute the power-of-two terms to the filter coefficients. Design examples show that the trade-off filters with discrete coefficients are less sensitive with respect to quantization than the least squares and minimax filters.

The paper is organized as follows. The problem formulation is discussed in Section II while the optimization approach is developed in Section III. The investigation of the filter with respect to quantization is discussed in Section IV. Design examples are given in Section $\mathrm{V}$ and finally concluding remarks are in Section VI.

\section{Problem Formulation}

Consider the design of a VFD filter with the desired frequency response given by

$$
\begin{gathered}
D(\omega, p)=e^{-j \omega p}=\cos (\omega p)-j \sin (\omega p), \\
|\omega| \leq \omega_{p}, p \in[-0.5,0.5] .
\end{gathered}
$$

The transfer function of the designed variable FIR filter is characterized by

$$
H(\omega, p)=\sum_{n=-N}^{N} h_{n}(p) e^{-j \omega n}
$$

where the coefficients $h_{n}(p)$ are expressed as polynomials in $p$ as

$$
h_{n}(p)=\sum_{m=0}^{M} a_{n, m} p^{m} .
$$


Thus,

$$
\begin{aligned}
H(\omega, p) & =\sum_{n=-N}^{N} \sum_{m=0}^{M} a_{n, m} e^{-j \omega n} p^{m} \\
& =\sum_{n=-N}^{N} \sum_{m=0}^{M_{c}} a_{n, 2 m} e^{-j \omega n} p^{2 m} \\
& +\sum_{n=-N}^{N} \sum_{m=1}^{M_{s}} a_{n, 2 m-1} e^{-j \omega n} p^{2 m-1}
\end{aligned}
$$

where

$$
\begin{cases}M_{c}=M_{s}=M / 2, & \text { for even } M \\ M_{c}+1=M_{s}=(M+1) / 2, & \text { for odd } M\end{cases}
$$

We employ symmetric properties for the coefficients $a_{n, m}$ as in [8], [9] to reduce the number of optimized coefficients

$$
a_{-n, m}= \begin{cases}a_{n, m}, & \text { for even } m \\ -a_{n, m}, & \text { for odd } m\end{cases}
$$

and $a_{n, 0}=\delta(n)$. The frequency response $H(\omega, p)$ can be reduced to

$$
\begin{aligned}
H(\omega, p) & =1+\sum_{m=1}^{M_{c}}\left(a_{0,2 m}+\sum_{n=1}^{N} 2 a_{n, 2 m} \cos (n \omega)\right) p^{2 m} \\
& -j \sum_{m=1}^{M_{s}}\left(\sum_{n=1}^{N} 2 a_{n, 2 m-1} \sin (n \omega)\right) p^{2 m-1} .
\end{aligned}
$$

Since

$$
H(\omega, p) \approx e^{-j \omega p}
$$

we have the following approximations

$$
1+\sum_{m=1}^{M_{c}}\left(a_{0,2 m}+\sum_{n=1}^{N} 2 a_{n, 2 m} \cos (n \omega)\right) p^{2 m} \approx \cos (\omega p)
$$

and

$$
\sum_{m=1}^{M_{s}}\left(\sum_{n=1}^{N} 2 a_{n, 2 m-1} \sin (n \omega)\right) p^{2 m-1} \approx \sin (\omega p) .
$$

Differentiating (5) with respect to $\omega$, we obtain

$$
\sum_{m=1}^{M_{c}} \sum_{n=1}^{N} 2 n a_{n, 2 m} \sin (n \omega) p^{2 m-1} \approx \sin (\omega p) .
$$

As such, there is a coefficient relationship for even $M, M_{c}=$ $M_{s},[8]$, namely

$$
a_{n, 2 m-1}=n a_{n, 2 m}, n=1, \ldots, N, m=1, \ldots, M_{c} .
$$

Thus, we have

$$
\begin{aligned}
H(\omega, p)= & 1+\sum_{m=1}^{M_{c}}\left[a_{0,2 m} p+2 \sum_{n=1}^{N} a_{n, 2 m}\right. \\
& \quad \begin{array}{c}
\cos (n \omega) p-j n \sin (n \omega))] p^{2 m-1} \\
=
\end{array}+\mathbf{c}^{T}(\omega, p) \mathbf{a}
\end{aligned}
$$

where $\mathbf{a}=\left[a_{0,2}, a_{1,2}, \ldots, a_{N, 2 M_{c}}\right]^{T}$,

$$
\begin{aligned}
\mathbf{c}(\omega, p)= & \mathbf{p} \otimes[p, 2 \cos (\omega) p-2 j \sin (\omega), \ldots, \\
& 2 \cos (N \omega) p-2 j N \sin (N \omega)]^{T} \\
\mathbf{p}= & {\left[p, p^{3}, \ldots, p^{2 M_{c}-1}\right]^{T} }
\end{aligned}
$$

and $\otimes$ denotes the Kronecker product.

\section{Optimization Problem}

The weighted integral squared error for the VFD filter can be expressed as

$$
e(\mathbf{a})=\int_{\Omega} \int_{\mathcal{P}} W(\omega, p)|H(\omega, p)-D(\omega, p)|^{2} d p d \omega
$$

where $W(\omega, p)$ is a positive weighting function, $\Omega=\left[0, \omega_{p}\right]$ and $\mathcal{P}=[-0.5,0.5]$. This error can be reduced to the quadratic form

$$
e(\mathbf{a})=\mathbf{a}^{T} \mathbf{G a}-2 \mathbf{p}^{T} \mathbf{a}+c
$$

where

$$
\begin{aligned}
\mathbf{G} & =\int_{\Omega} \int_{\mathcal{P}} W(\omega, p) \mathcal{R}\left(\mathbf{c}(\omega, p) \mathbf{c}^{H}(\omega, p)\right) d p d \omega \\
\mathbf{p} & =\int_{\Omega} \int_{\mathcal{P}} W(\omega, p) \mathcal{R}\left(\mathbf{c}(\omega, p) D^{*}(\omega, p)\right) d p d \omega \\
c & =\int_{\Omega} \int_{\mathcal{P}} W(\omega, p)|D(\omega, p)|^{2} d p d \omega .
\end{aligned}
$$

Here "*" and $\mathcal{R}(\cdot)$ denote the conjugate and the real part of a complex number, respectively. The weighted least squares solution is $\mathbf{a}_{L S}=\mathbf{G}^{-1} \mathbf{p}$.

The least squares solution in general has a low integral squared error and a large peak deviation error. The minimax solution, on the other hand, often has low peak deviation error and a large integral squared error. For the VFD filter with coefficient symmetry, the minimax optimization problem can be formulated as:

$$
\left\{\begin{array}{l}
\min _{\epsilon, \mathbf{a}} \epsilon \\
W(\omega, p)|H(\omega, p)-D(\omega, p)| \leq \epsilon, \forall \omega \in \Omega, p \in \mathcal{P} .
\end{array}\right.
$$

This problem can be equivalently written as

$$
\left\{\begin{array}{l}
\min _{\epsilon, \mathbf{a}} \epsilon \\
W(\omega, p) \sqrt{e_{R}^{2}(\omega, p)+e_{I}^{2}(\omega, p)} \leq \epsilon, \forall \omega \in \Omega, p \in \mathcal{P}
\end{array}\right.
$$

where

$$
\begin{aligned}
e_{R}(\omega, p) & =\mathbf{c}_{R}^{T}(\omega, p) \mathbf{a}+1-\cos (\omega p) \\
e_{I}(\omega, p) & =\mathbf{c}_{I}^{T}(\omega, p) \mathbf{a}+\sin (\omega p)
\end{aligned}
$$

and $\mathbf{c}_{R}(\omega, p), \mathbf{c}_{I}(\omega, p)$ denote, respectively, the real and the imaginary parts of $\mathbf{c}(\omega, p)$. The optimization (13) is equivalent to

$$
\left\{\begin{array}{l}
\min _{\epsilon, \mathbf{a}} \epsilon \\
\left(\epsilon,\left[\begin{array}{l}
W(\omega, p) e_{R}(\omega, p) \\
W(\omega, p) e_{I}(\omega, p)
\end{array}\right]\right) \in \mathrm{Q}_{\text {cone }}, \forall \omega \in \Omega, p \in \mathcal{P}
\end{array}\right.
$$


where the quadratic cone is defined as

$$
\mathrm{Q}_{\text {cone }}:=\left\{(\epsilon, \mathbf{x}) \in \mathcal{R} \times \mathcal{R}^{I}: \epsilon \geq\|\mathbf{x}\|\right\}
$$

and $I$ is the dimension of $\mathbf{x}$ and $\|\cdot\|$ is the Euclidean norm. The problem (15) is an SOCP which can be solved efficiently using SOCP software such as SeDuMi [11].

Now, we investigate the trade-off between the integral squared error and the peak deviation error by incorporating constraints on the peak deviation error. The optimization problem can be formulated as

$$
\left\{\begin{array}{l}
\min _{\mathbf{a}} e(\mathbf{a})=\mathbf{a}^{T} \mathbf{G a}-2 \mathbf{p}^{T} \mathbf{a}+c \\
W(\omega, p)|H(\omega, p)-D(\omega, p)| \leq \alpha, \forall \omega \in \Omega, p \in \mathcal{P}
\end{array}\right.
$$

where $\alpha$ is the upper bound for the frequency response deviation for all $\omega$ and $p$. For (16) to have a feasible solution, $\alpha$ is chosen such that $\alpha \geq \epsilon_{M M}$, where $\epsilon_{M M}$ is the peak deviation error for the minimax optimization problem (15). Since the matrix $\mathbf{G}$ is symmetric and positive definite, we can do a Cholesky factorization of $\mathbf{G}$ as the product of a lower triangular matrix $\mathbf{R}$ and its transpose

$$
\mathbf{G}=\mathbf{R}^{T} \mathbf{R} \text {. }
$$

Let $\tilde{\mathbf{p}}=\left(\mathbf{R}^{T}\right)^{-1} \mathbf{p}$, then the integral squared error $e(\mathbf{a})$ in (16) can be expressed as

$$
e(\mathbf{a})=\|\mathbf{R a}-\tilde{\mathbf{p}}\|^{2}-\mathbf{p}^{T} \mathbf{G}^{-1} \mathbf{p}+c .
$$

Since the term $-\mathbf{p}^{T} \mathbf{G}^{-1} \mathbf{p}+c$ is a constant, minimizing $e(\mathbf{a})$ is equivalent to minimizing the norm $\|\mathbf{R a}-\tilde{\mathbf{p}}\|$. As such, the problem (16) can be reformulated as:

$$
\left\{\begin{array}{l}
\min _{\gamma, \mathbf{a}} \gamma \\
\|\mathbf{R a}-\tilde{\mathbf{p}}\| \leq \gamma \\
W(\omega, p) \sqrt{e_{R}^{2}(\omega, p)+e_{I}^{2}(\omega, p)} \leq \alpha, \forall \omega \in \Omega, p \in \mathcal{P} .
\end{array}\right.
$$

The problem (19) is solved by using discretization. Denote by $N_{\omega}$ and $N_{p}$ and the number of discretized points for $\omega$ and $p$, respectively. Let $\mathbf{x}=[\gamma ; \mathbf{a}]$, then (19) can be expressed in the standard SOCP formulation as

$$
\left\{\begin{array}{l}
\min _{\mathbf{x}} \mathbf{b}^{T} \mathbf{x} \\
\left\|\mathbf{A}_{0} \mathbf{x}-\tilde{\mathbf{p}}\right\| \leq \mathbf{b}^{T} \mathbf{x} \\
\left\|\mathbf{A}_{i, k} \mathbf{x}+\mathbf{c}_{i, k}\right\| \leq \alpha, \forall 1 \leq i \leq N_{\omega}, 1 \leq k \leq N_{p} .
\end{array}\right.
$$

where $\mathbf{b}=\left[\begin{array}{ll}1,0, \ldots, 0\end{array}\right]^{T}, \mathbf{A}_{0}=\left[\begin{array}{ll}\mathbf{0} & \mathbf{R}\end{array}\right]$,

$$
\begin{aligned}
\mathbf{A}_{i, k} & =\left[\begin{array}{cc}
0 & W\left(\omega_{i}, p_{k}\right) \mathbf{c}_{R}^{T}\left(\omega_{i}, p_{k}\right) \\
0 & W\left(\omega_{i}, p_{k}\right) \mathbf{c}_{I}^{T}\left(\omega_{i}, p_{k}\right)
\end{array}\right] \\
\mathbf{c}_{i, k} & =\left[\begin{array}{c}
W\left(\omega_{i}, p_{k}\right)\left(1-\cos \left(\omega_{i}, p_{k}\right)\right) \\
W\left(\omega_{i}, p_{k}\right) \sin \left(\omega_{i}, p_{k}\right)
\end{array}\right] .
\end{aligned}
$$

Alternatively, the problem (19) can be written as

$$
\left\{\begin{array}{l}
\min _{\gamma, \mathbf{a}} \gamma \\
(\gamma, \mathbf{R a}-\tilde{\mathbf{p}}) \in \mathrm{Q}_{\text {cone }} \\
\left(\alpha,\left[\begin{array}{c}
W\left(\omega_{i}, p_{k}\right) e_{R}\left(\omega_{i}, p_{k}\right) \\
W\left(\omega_{i}, p_{k}\right) e_{I}\left(\omega_{i}, p_{k}\right)
\end{array}\right]\right) \in \mathrm{Q}_{\text {cone }} \\
\forall 1 \leq i \leq N_{\omega}, 1 \leq k \leq N_{p} .
\end{array}\right.
$$

The optimization problem (21) can be solved accurately using SOCP software such as SeDuMi. The computational complexity for solving the problem is similar to the minimax problem (14), [9]-[10]. In the following, we investigate the sensitivity of (i) the least squares solution; (ii) the minimax solution and (iii) the trade-off solutions with the sum of signed power-of-two (SOSPT) coefficients to reduce operations associated with the filters to shifts and adds.

\section{Quantization Performance}

To investigate the performance of VFD filter with discrete coefficients, the VFD filter coefficient $a_{n, m}$ are stacked for even and odd $m$ in a coefficient vector $\mathbf{h}$ as

$$
\mathbf{h}=\left[\mathbf{a}, a_{1,1}, \cdots, a_{N, 2 M_{c}-1}\right] .
$$

The number of coefficients in $\mathbf{h}$ is $M_{c}(1+2 N)=2 N M_{c}+$ $M_{c}$. Denote by $\mathbf{h}_{q}$ the quantized coefficient vector for $\mathbf{h}$. For all $1 \leq k \leq 2 N M_{c}+M_{c}$, denote by $c_{k}$ the number of power-of-two terms in the coefficient $h_{q}(k)$. Then, the coefficient $h_{q}(k)$ can be expressed as SOSPT terms $h_{q}(k)=$ $\sum_{k=1}^{c_{k}} \eta_{k} 2^{-\nu_{k}}$ where $\eta_{k}$ is a binary value, $\eta_{k} \in\{-1,1\}$ and $\nu_{k}$ is an integer, $B_{\ell} \leq \nu_{k} \leq B_{u}$. Here, the values $B_{\ell}$ and $B_{u}$ denote the maximum and minimum value for the power-oftwo. The total number of power-of-two terms for the VFD is restricted to a positive number $L$ according to

$$
\sum_{k=1}^{2 N} c_{c}+M_{c} \leq L
$$

Since each filter coefficient is not restricted to a fixed number of power-of-two terms, there is a degree of freedom in distributing the power-of-two terms to the appropriate VFD coefficients. We employ the quantization approach [12] to the VFD filter coefficients with the infinite precision solution $\mathbf{h}$. The quantization procedure is summarized as follows.

Procedure IV.1: Quantization procedure for $\mathbf{h}$ given an upper bound $L$ on the total number of power-of-two terms. - Step 1: Initialize $\mathbf{h}_{q}$ as a zero vector and set $k=1$.

- Step 2: Search for an index $\ell, 1 \leq \ell \leq 2 N M_{c}+M_{c}$, corresponding to the coefficient in $\mathbf{h}$ with the maximum absolute value, $|h(n)|=\max _{1 \leq \ell \leq 2 N M_{c}+M_{c}}|h(\ell)|$. We have the following two cases: If $|h(n)|<2^{-B_{u}-1}$, then stop the procedure. Otherwise, search for a power-of-two term that is closest to $h(n)$,

$$
\left|h(n)-\zeta_{1}\right|=\min _{\zeta \in \mathcal{B}}|h(n)-\zeta|
$$

where $\mathcal{B}=\left\{-2^{-B_{u}}, \ldots,-2^{-B_{l}}, 2^{-B_{l}}, \ldots, 2^{-B_{u}}\right\}$. Locate the $k^{\text {th }}$ power-of-two term to the $n^{\text {th }}$ position of $\mathbf{h}_{q}$. Update the quantized vector $\mathbf{h}_{q}$ by adding $\zeta_{1}$ to the coefficient $h_{q}(n)$ and update $\mathbf{h}$ by subtracting $\zeta_{1}$ from $h(n)$. If $k<L$, then set $k:=k+1$ and return to the beginning of Step 2 . Otherwise, stop the procedure. The vector $\mathbf{h}_{q}$ is the quantized solution with a restriction of $L$ on the total number of power-of-two terms. 


\section{Design Examples}

Consider the design of variable fractional delay FIR filter with $\omega_{p}=0.9 \pi$. The range for $p$ is $[-0.5,0.5]$. The number of discretization points for $\omega$ is $L_{\omega}=512$, whereas the number of discretization points for $p$ is $L_{p}=128$. As in [7]-[10], the weighting function $W(\omega, p)$ is chosen as one for all $\omega$ and $p$.

Fig. 1 shows the trade-off curve between the peak deviation error and the integral squared error for the case $N=20, M=6$ and $N=25, M=6$. The least squares and the minimax solutions are at the two extreme points of the curves. As can be seen from the figure, it is possible to reduce the peak deviation error by $6.4 \mathrm{~dB}$ from the least squares solution with just a $0.6 \mathrm{~dB}$ increase in the integral squared error. Similarly, it is possible to reduce the integral squared error by $3 \mathrm{~dB}$ from the minimax solution with just an increase of $0.3 \mathrm{~dB}$ in the peak deviation error.
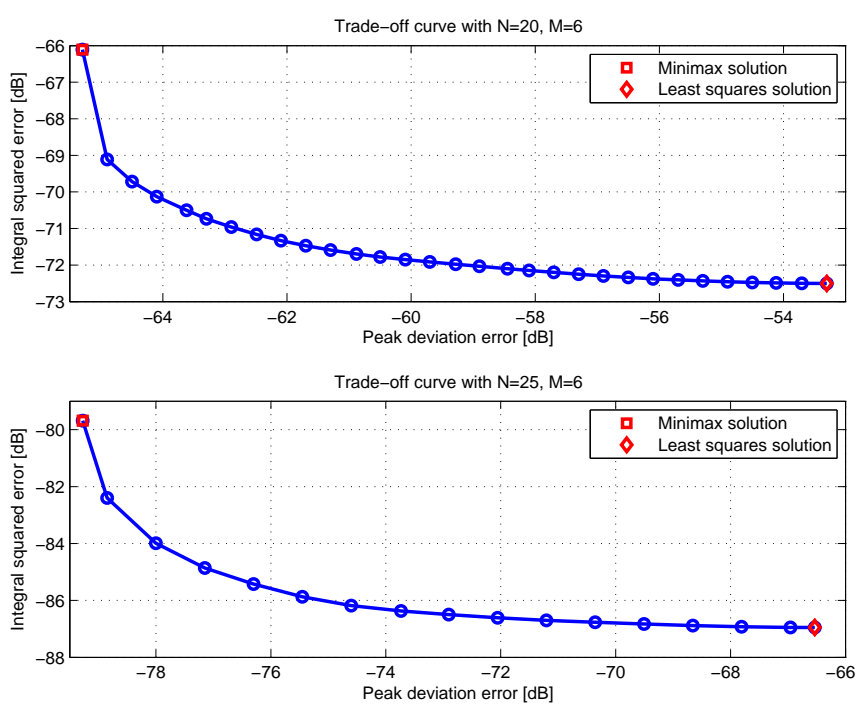

Fig. 1. Trade-off between the peak deviation error and the integral squared error for $N=20, M=6$ and $N=25, M=6$.

Table I shows (i) the integral squared error $e_{2}$; (ii) the minimax error $e_{\max }$; (iii) the maximum magnitude response error $e_{m, m a g}$; and (iv) the maximum group delay error $e_{m, g d}$ for the least squares and minimax solutions. The table also shows the performance of two VFD filters in the trade-off curve. As can be seen from the table, the magnitude response error and group delay error for the trade-off solution 1 are significantly lower than for the least squares solution while the errors for the trade-off solution 2 are approximately the same as the minimax solution. The trade-off solution 2, on the other hand, is approximately $3 \mathrm{~dB}$ lower in the integral squared error than the minimax solution.

Figs. 2 and 3 show the trade-off between the integral squared error and the peak deviation error when $N$ increases from 30 to 35 . As with the previous case, the peak error deviation can be reduced significantly from the least squares solution with a small increase in the integral squared error. In addition, the integral squared error can
TABLE I

Magnitude And group Delay ERrors with $N=25$ and $M=6$.

\begin{tabular}{|c|c|c|c|c|}
\hline Solutions & $\begin{array}{c}e_{2} \\
{[\mathrm{~dB}]}\end{array}$ & $\begin{array}{c}\epsilon_{\max } \\
{[\mathrm{dB}]}\end{array}$ & $\begin{array}{c}e_{m, \operatorname{mag}} \\
{[\mathrm{dB}]}\end{array}$ & $\begin{array}{c}\epsilon_{m, g d} \\
{[\mathrm{~dB}]}\end{array}$ \\
\hline Least squares & -86.95 & -66.53 & -66.97 & -32.40 \\
\hline Trade-off sol. 1 & -86.55 & -72.48 & -72.69 & -33.22 \\
\hline Minimax & -79.69 & -79.27 & -79.42 & -40.85 \\
\hline Trade-off sol. 2 & -82.40 & -78.85 & -79.00 & -39.10 \\
\hline
\end{tabular}

be reduced significantly from the minimax solution with a small increase in the peak deviation error.
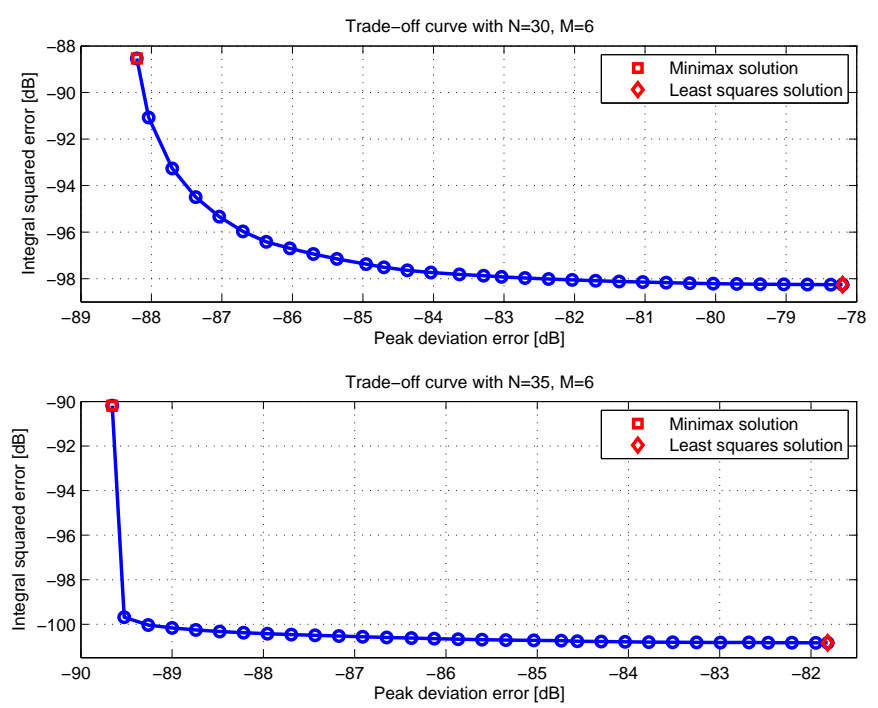

Fig. 2. Trade-off between the peak deviation error and the integral squared error for $N=30, M=6$ and $N=35, M=6$.

Table II shows the quantization performance of the least squared, the minimax solutions and two VFD filters in the trade-off curve for $N=20$ and $M=6$. As the maximum coefficient for the infinite precision solution is approximately 1 and the peak deviation error for the minimax solution is $-65 \mathrm{~dB}, B_{\ell}$ and $B_{u}$ are chosen as $B_{\ell}=0$ and $B_{u}=13$. The total number of power-of-two for the VFD filters increases from $2.5 M N$ to $3.5 M N$. For $L=3.5 M N$, the quantized least squares solution is $0.3 \mathrm{~dB}$ lower in integral squared error than the trade-off solution 1 with $6.6 \mathrm{~dB}$ higher in the peak deviation error. Also, the quantized minimax solution is $0.75 \mathrm{~dB}$ lower in the peak deviation error than the trade-off solution 2 and $2.2 \mathrm{~dB}$ higher in the integral squared error. For lower values of $L$, the trade-off filters are less sensitive with respect to quantization than the least squares and minimax filters. This is due to the fact that the trade-off solutions have low integral squared error together with low peak deviation error.

Table III shows the quantization performance of the least squares, the minimax and the trade-off solutions for $N=25$ and $M=6$. The table also shows the effect of quantization when $b_{u}$ increases from 13 to 15 . Similar to the previous case, the trade-off solutions are less sensitive with respect to the quantization than the least squares and 


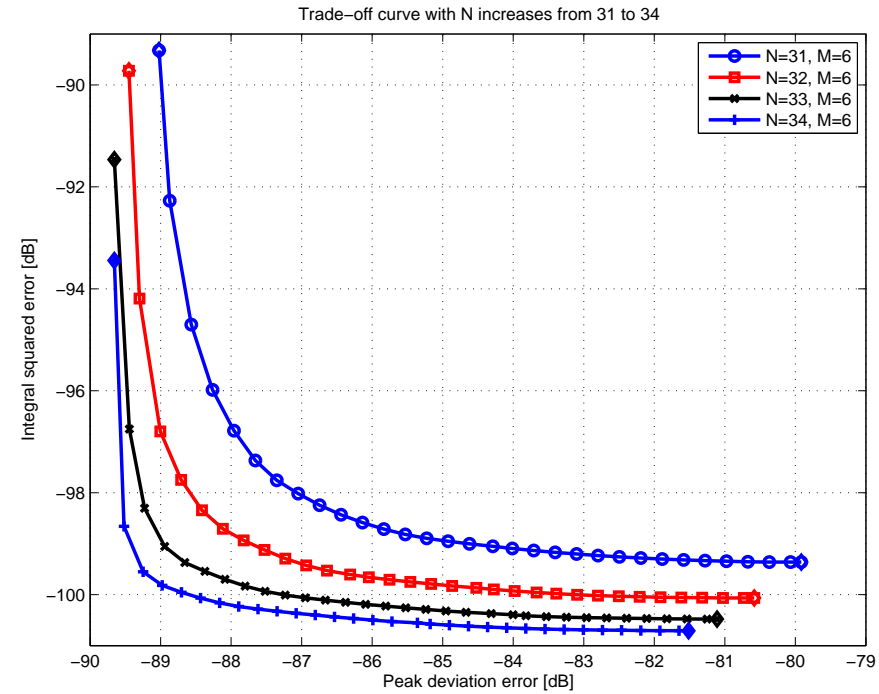

Fig. 3. Trade-off between the peak deviation error and the integral squared error with $N$ increases from 31 to 34 .

TABLE II

EfFeCt Of QUANTIZATION With $N=20, M=6$ and $b_{u}=13$.

\begin{tabular}{|c|c|c|c|}
\hline & Solutions & $e_{2}[\mathrm{~dB}]$ & $e_{m}[\mathrm{~dB}]$ \\
\hline \multirow{4}{*}{ Least } & Inf. precision & -72.50 & -53.30 \\
\cline { 2 - 4 } squares & $L=2.5 M N$ & -65.72 & -52.62 \\
& $L=3 M N$ & -70.04 & -52.68 \\
& $L=3.5 M N$ & -71.37 & -53.02 \\
\hline \multirow{3}{*}{ Trade-off } & Inf. precision & -71.92 & -59.70 \\
\cline { 2 - 4 } solution & $L=2.5 M N$ & -67.10 & -56.95 \\
1 & $L=3 M N$ & -70.75 & -58.71 \\
\hline \multirow{3}{*}{ Minimax } & $L=3.5 M N$ & -71.07 & -59.62 \\
\cline { 2 - 4 } & Inf. precision & -66.10 & -65.29 \\
\cline { 2 - 4 } & $L=2.5 M N$ & -61.26 & -54.08 \\
Trade-off & $L=3 M N$ & -65.10 & -59.80 \\
solution & $L=3.5 M N$ & -66.10 & -62.77 \\
\cline { 2 - 4 } 2 & $L=2.5 M N$ & -66.54 & -64.90 \\
\hline & $L=3 M N$ & -68.01 & -61.75 \\
& $L=3.5 M N$ & -68.30 & -62.02 \\
\hline
\end{tabular}

the minimax solutions.

\section{Conclusions}

This paper investigates a trade-off between the integral squared error and the peak deviation error for the VFD filter with coefficient relationship. The integral squared error is minimized subject to additional constraints on peak deviation error. Design examples show that the peak deviation error can be reduced significantly from the least squares solution while maintaining approximately the same integral squared error. Also, the integral squared error can be reduced significantly from the minimax solution while maintaining approximately the same peak deviation error. In addition, the trade-off filters are less sensitive with respect to quantization than the least squares and minimax filters.
TABLE III

Effect of QUANTIZATION With $N=25$ and $M=6$.

\begin{tabular}{|c|c|c|c|}
\hline & Solutions & $e_{2}[\mathrm{~dB}]$ & $e_{m}[\mathrm{~dB}]$ \\
\hline \multirow{4}{*}{ Least } & Inf. precision & -86.95 & -66.53 \\
\cline { 2 - 4 } squares & $L=2.5 M N, b_{u}=13$ & -69.59 & -61.81 \\
& $L=3 M N, b_{u}=13$ & -78.85 & -65.84 \\
& $L=3 M N, b_{u}=15$ & -84.78 & -66.86 \\
\hline \multirow{3}{*}{ Trade-off } & $L=2.5 M N, b_{u}=13$ & -74.30 & -72.48 \\
\cline { 2 - 4 } solution & $L=3 M N, b_{u}=13$ & -78.10 & -70.55 \\
1 & $L=3 M N, b_{u}=15$ & -85.00 & -70.55 \\
\hline \multirow{3}{*}{ Minimax } & Inf. precision & -79.69 & -79.27 \\
\cline { 2 - 4 } & $L=2.5 M N, b_{u}=13$ & -65.86 & -58.75 \\
& $L=3 M N, b_{u}=13$ & -74.46 & -66.94 \\
& $L=3 M N, b_{u}=15$ & -78.71 & -74.11 \\
\hline \multirow{3}{*}{ Trade-off } & $L=2.5 M N, b_{u}=13$ & -82.40 & -78.85 \\
solution & $L=3 M N, b_{u}=13$ & -76.20 & -60.64 \\
2 & $L=3 M N, b_{u}=15$ & -80.72 & -68.13 \\
\cline { 2 - 4 } 2 & $I n f . \operatorname{precision}$ & -73.73 \\
\hline
\end{tabular}

\section{REFERENCES}

[1] C. W. Farrow, "A continuously variable digital delay element," in Proc. IEEE Int. Symp. Circuits Syst., vol. 3, pp. 2641-2645, Jun. 1988.

[2] H. H. Dam, A. Cantoni, K. L. Teo, S. Nordholm, "Variable digital filter with least square criterion and peak gain constraints," IEEE Trans. Circuits Systems II, vol. 54, no. 1, pp. 24-28, Jan. 2007.

[3] H. H. Dam, A. Cantoni, K. L. Teo, and S. Nordholm, "Variable digital filter with group delay flatness specification or phase constraints," IEEE Trans. Circuits Systems II, vol. 55, no. 5, pp. 442-446, May 2008.

[4] H. H. Dam, "Variable fractional delay filter with sub-expressions coefficients," International Journal of Innovative Computing, Information and Control, vol. 9, no. 7, pp. 2995-3003, Jul. 2013.

[5] H. H. Dam, "Design of Allpass Variable Fractional Delay Filter," IEEE Transactions on Signal Processing, vol. 59, no. 12, pp. 6240-6244, Dec. 2011.

[6] C.-C. Tseng, "Design of variable fractional delay FIR filters using symmetry," in Proc. IEEE Int. Symp. Circuits Syst," vol. III, pp. 477-480, May 2004.

[7] T.-B. Deng and Y. Lian, "Weighted-least-squares design of variable fractional-delay FIR filters using coefficient symmetry," IEEE Trans. Signal Process., vol. 54, no. 8, pp. 3023-3038, Aug. 2006.

[8] Y.-D. Huang, S.-C. Pei, and J.-J. Shyu, "WLS design of variable fractional-delay FIR filters using coefficient relationship," IEEE Trans. Circuits Systems II: Express Brief, vol. 56, no. 3., pp. 220-224, Mar. 2009.

[9] T.-B. Deng and S. Chivapreecha, "Bi-minimax design of evenorder variable fractional-delay FIR digital filters," IEEE Trans. Circuits Systems I: Reg. Paper, vol. 59, no. 8, pp. 1766-1774, Aug. 2012.

[10] T.-B. Deng and W. Qin, "Coefficient relation-based minimax design and low-complexity structure of variable fractional-delay digital filters," Signal Processing, vol. 93, pp. 923-932, Apr. 2013.

[11] J. F. Sturm, "Using SeDuMi 1.02, a MATLAB toolbox for optimization over symmetric cones," Optimization Methods and Software 11-12 (1999) 625-653. Special issue on Interior Point Methods (CD supplement with software).

[12] D. Li, Y. C. Lim, Y. Lian, and J. Song, "A polynomial-time algorithm for designing FIR filters with power-of-two coefficients," IEEE Trans. Signal Procc., vol. 50, no. 8, pp. 1935-1940, Aug. 2002

[13] C. K. S. Pun, Y. C. Wu, S. C. Chan, and K. L. Ho, "On the design and efficient implementation of the Farrow structure," IEEE Signal Processing Letters, vol. 10, no. 7, pp. 189-192, Jul. 2003 